\title{
Decision Support Framework for PLM Harmonization Projects within Industrial Companies
}

\author{
Michael Abramovici and Jens Christian Göbel \\ Chair of Information Technology in Mechanical Engineering (ITM), \\ Bochum Ruhr-University, Bochum, Germany \\ \{michael.abramovici, jenschristian.goebel\}@itm.rub.de
}

\begin{abstract}
To this day, as a result of increasing PLM penetration and continuously changing company structures, several PLM solutions have evolved over time and now exist in parallel within many companies. Disharmonized PLM solutions can impede productivity and flexibility in cross-division cooperation and company-internal reorganization projects. A lot of companies carry out PLM harmonization projects with the goal of improving their current situation due to an optimal coordination, adaptation, and standardization of their existing PLM solutions. The present paper introduces a comprehensive framework which supports fundamental decisions that have to be made in this context. This framework allows an objectified determination of harmonization concepts by using both directly and non-directly monetarily quantifiable measures taking into account the perspectives of all of the actors involved. This approach emerged from the experiences gained from several industrial use cases, including the one that is illustrated in this paper.
\end{abstract}

Keywords: PLM harmonization, PLM planning, PLM integration, PLM procedure model, PLM decision framework.

\section{$1 \quad$ Current Situation}

Over the past two decades, the penetration of Product Lifecycle Management (PLM) in industrial companies has constantly increased. This applies to the different engineering disciplines (mechanics, electronics, software, hydraulics, etc.) as well as to the different product lifecycle phases downstream the product development (production planning, services, etc.) [1], [12]. To this day, as a result of increasing PLM penetration, several PLM solutions have evolved and now exist in parallel in many companies. These historically grown solutions are, for the most part, very strongly oriented towards division-specific requirements but they have not or only partially been coordinated. In addition to this organic evolution within the companies, mergers and acquisitions of companies or company divisions escalate PLM heterogeneity. This heterogeneity is mainly evident from the large number of PLM IT systems employed [1], but can equally concern PLM strategy-, PLM process-, PLM method-, and PLM actor-related components [2], [15]. The unplanned heterogeneity in industrial PLM landscapes is permanently increasing within globally distributed and continuously changing engineering organization structures [4]. Hence, disharmonized 
PLM solutions can impede productivity and flexibility in cross-division cooperation or company-internal reorganization projects. By carrying out PLM harmonization projects, companies pursue the goal of improving their current situation by means of suitable coordination, adaptation, and standardization of existing PLM solutions. As an example, the harmonization of several company divisions may concern the mutually used part names or part numbers exclusively without requiring changes of the used PLM systems.

In most cases, neither an exhaustive company-wide PLM standardization, nor retaining the heterogeneous, current state provide an optimal solution to a PLM harmonization that is oriented towards company-specific requirements. Instead, a harmonization concept geared to strategic, organizational, and economic goals has to be developed, which must be based on a simultaneous and thus complex detail consideration of any of the involved PLM solutions. This task involves fundamental harmonization decisions that should bring out the optimal harmonization concept. These harmonization decisions directly determine the scale of required changes to the existing PLM solutions. They are characterized by

- high complexity due to the diversity of the involved company divisions, PLM components, lifecycle phases, and possible concept alternatives,

- diverging individual interests and targets of formal and informal actors with a broad range of different personal experiences, domain knowledge, and subjective preferences,

- far-reaching and difficult-to-predict consequences that may lead to severe repercussions on the project and corporate success, and

- ambiguous decision-making structures within the company due to the scarcity of occurrence and a lack of awareness.

Making these harmonization decisions faces PLM decision makers and planners with challenges that are difficult to overcome. Nowadays, such decisions are usually made without any methodical support. Thus, the pursued harmonization concept highly depends on subjective preferences, political influences, informal power structures, and the assertiveness of the individual actors. Poorly substantiated harmonization decisions often lead to consequences of which decision makers are only partially aware (e.g. costs or risks) and that are not accepted and supported by the majority of PLM actors (e.g. users).

\section{Requirements}

In order to prevent these problems and as a basis for successful PLM harmonization initiatives, a suitable Harmonization Decision Framework (HDF) is mandatory. This HDF must enable PLM decision makers and planners to make methodical and objective decisions that are consequently geared towards top-level targets of the company through

- simultaneous consideration of the design of several PLM solutions,

- holistic consideration of the PLM solutions to be harmonized, i.e. the PLM levels of PLM strategy, PLM processes, PLM methods, PLM IT systems (data included) and PLM actors (PLM users, several PLM specialists, PLM planners, PLMrelevant decision makers and project sponsors) [2], 
- identification and demarcation of harmonization decision-relevant components of all PLM solutions considered,

- systematic consideration of interactions between PLM components to be actively harmonized and PLM components influenced by this procedure,

- generic focus on any PLM harmonization components that are to be addressed use case-specifically,

- scalability and adaptability to support company-specific harmonization decision problems,

- objective alignment with company-wide and division-specific targets and constraints,

- consideration of the perspectives and subjective preferences of the decision makers in a balanced target system,

- prospective evaluation of direct and indirect monetary effects of harmonization concept alternatives on all PLM levels,

- flexible applicability combined with different PLM procedure models,

- practicability with regard to the resources required for the application of the HDF (personalized skills, knowledge and experiences, additional information provided, expenses and duration of the application).

\section{$3 \quad$ Related Methods and Works}

The HDF addresses a methodical support that is required for PLM harmonization projects. In the course of the establishment of PLM and its previous evolutionary phases, in particular Product Data Management (PDM), a multitude of procedure models for methodical support of PLM projects has been developed. The most important works related to this paper are presented and briefly discussed in the following:

The VDI 2219 guideline published by the Association of German Engineers (VDI) focuses on the introduction and economics of EDM/PDM systems [17]. It can be considered a reference to the scope of the project phases to cover a classic introduction of a new EDM/PDM system. In this context, EDM/PDM system selection decisions are methodically supported. VDI 2219 does not address decision problems that emerge within the scope of the simultaneous design of several existing PLM solutions. Moreover, it strongly focuses on IT systems. PDM and PLM system selection decisions are also addressed in works by [3], [8] and [10].

The German Machine and Plant Engineering Association (VDMA) has developed a guideline for the creation of company-specific PLM concepts that focuses on PLM introduction and extension whilst taking the existing PLM components into account [18]. From the elaborated PLM concept, a decision paper, i.e. the solution space confined to the implementation and non-implementation of the concept, has been derived. This guideline, however, does not address the fact that there are several existing PLM solutions and concept alternatives for their design.

Within the scope of transfer area No. 57, funded by Deutsche Forschungsgemeinschaft (DFG), a guideline for the evaluation and selection of system-independent PLM reference processes has been developed [14]. The maturity level-based evaluation method elaborated for this purpose focuses on the evaluation of improvement 
potentials of company-specific current state processes via the developed PLM reference processes. The evaluation method is primarily geared to PLM processes and therefore does not provide any instruments for an integrated evaluation of PLM harmonization concept alternatives.

Furthermore, there are several IT-related PLM standards that support the exchange and management of product data. One of the most important ones is the 'Standard for the Exchange of Product model data' (STEP) released by the International Standard Organization [11]. As a part of STEP, application protocol 239 that provides a generic 'Product Lifecycle Support' (PLCS) [7] can be particularly relevant with regard to special information technical aspects when modeling PLM harmonization concept alternatives.

The procedure model developed by Trippner aims at the creation of the prerequisites for a continuous control of product data management in complex and dynamic product development processes [16]. This PDM system-centered approach is based on continuous organizational structuring of companies into PDM fractals and PDM central areas. In this case, only escalation options are mentioned whereas methodical planning of decisions on competing alternatives for a design of several PLM solutions is not in the focus of the procedure model.

To start with, in his empirical study "Do IT Smart", Höfener has developed a method for the evaluation of the strategic benefit of integrated product data management [6]. This method aims at supporting a decision for or against PLM investments by prospectively evaluating the benefit of the PLM system, with respect to the typology of the company in question. It is strongly IT system-oriented and also considers impacts on processual and strategic targets that are difficult to quantify. Höfener's approach, however, does not support cross-division harmonization concept decisions for heterogeneous PLM solutions within a company.

Schabacker describes a method for the evaluation of company-specific PLM benefits that is based on a model of the finance portfolio theory [13]. This method considers both directly and non-directly monetarily quantifiable PLM benefits. The availability of mathematical functions that describe experiences concerning benefits and risks in analytic terms, however, is mandatory for the applicability of the method. The requirements for a Harmonization Decision Framework as described above are only partially met by the method suggested by Schabacker, particularly with respect to flexibility and practicability.

Thus, existing methods and models for the support of PLM projects emphasize the field of introducing and optimizing singular PLM solutions as well as the decision on the PLM system to be applied, which is often central in this context. The PLM harmonization project requirements for a methodical support of harmonization concept decisions are, however, not met by these solutions.

\section{PLM Harmonization Decision Framework (HDF)}

This section introduces the HDF, a generic methodical approach that allows the capturing, description, and systematic reduction of the design space of decision problems 
that occur in PLM harmonization projects in a formal and transparent way. The aim of the HDF is to provide a use case-configurable and adaptable tool for objectified determination of optimal harmonization concept alternatives for an individual company. The formulation and evaluation of the concept alternatives ensues from a crossdivision perspective and takes into account the knowledge and experiences of all actors involved. The HDF comprises four successive stages (cf. fig. 1).

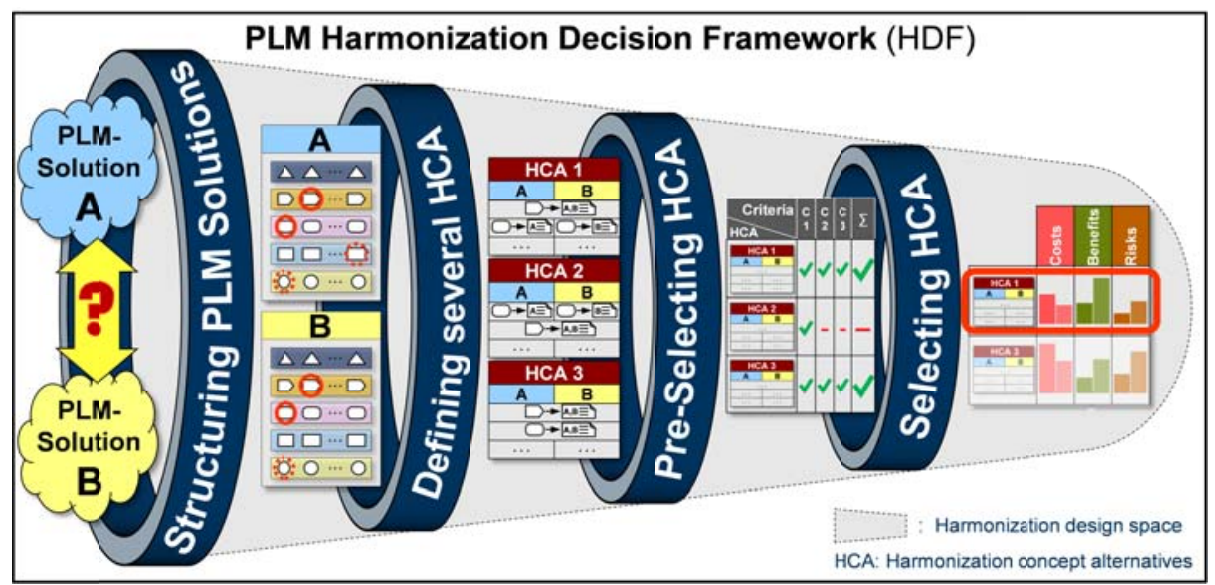

Fig. 1. Overview of the Harmonization Decision Framework (HDF)

\subsection{Structuring PLM Solutions}

A systems theoretical consideration of PLM solutions serves as a basis for their capturing and structuring. Thus, company-specific PLM solutions can generally comprise components of the five PLM levels: PLM strategy, PLM processes, PLM methods, PLM systems and data, as well as PLM actors (cf. figure 2). Every PLM level is delineated by several concrete PLM components that can have interdependencies with other components. Corresponding to the diversity of the PLM levels, the spectrum of possible PLM components is very broad. As an example, the expertise of a user on a PLM method and the PLM vendor strategy can equally constitute components of a PLM solution. With the aid of project-neutral templates, each of the considered PLM solutions is to be defined and demarcated with regard to their scope and focus areas by means of concrete PLM components and in a consistent manner.

Based on this, the entirety of the identified PLM components can be classified into actively focused or passively affected PLM components according to their role in the respective harmonization project. The application of the proposed systematization realizes a structured, transparent overview of all relevant PLM components to be dealt with. 


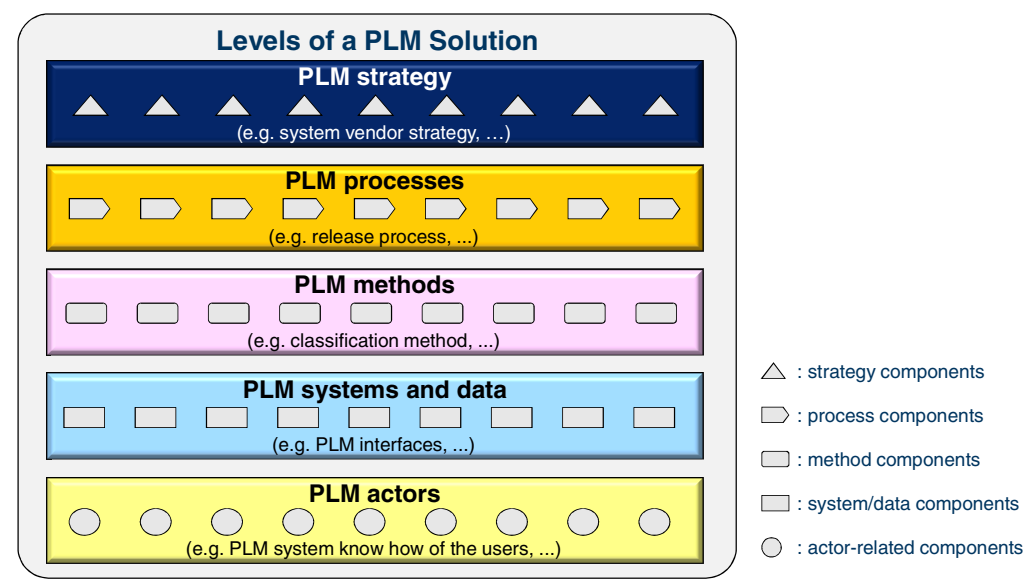

Fig. 2. Systematization for the structuring of PLM solutions

\subsection{Defining Harmonization Concept Alternatives}

The term 'harmonization concept alternative' refers to a combination of formalized variants of the PLM components to be actively focused, which, in turn, defines a single comprehensive alternative for the harmonization task at hand. This includes PLM components of all company divisions concerned. Three basic harmonization approaches are distinguished to define these concept alternatives, each of which features different degrees of harmonization (cf. figure 3).

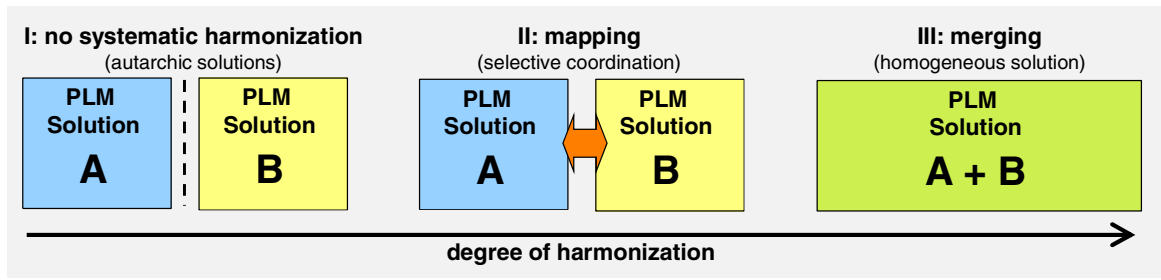

Fig. 3. Overview of the basic harmonization approaches

Overall standards and restrictions for the scope of the design of the focused PLM components are defined by means of basic harmonization approaches. These basic harmonization approaches can be defined on three aggregation levels, either for individual PLM components, for groups of PLM components, or for entire PLM levels (and all PLM components included). The definition of permitted harmonization approaches includes the representatives of any involved division and has to be backed by the overall-responsible PLM decision makers. Based on an exhaustive definition of all admissible basic harmonization approaches for every PLM component, at least one 
variant has to be defined for every admissible combination of a PLM component and its respective approaches. Suitable methods and tools, in turn, should support the definition of the concrete variants. The selection and implementation of appropriate modeling methods and tools (e.g. process and data modeling methods) is PLM component-specific and does not constitute the focus of the HDF. These activities should be covered by the applied project procedure model [2].

\subsection{Pre-selecting Harmonization Concept Alternatives}

The pre-selecting in advance of the detailed evaluation serves the elimination of harmonization concept alternatives that do not conform to particular restrictions or specifications. A catalog of exclusion criteria for each PLM level forms the basis for this. These criteria define strategic requirements for the concept alternative to be pursued, the compliance of which has to be ensured by all means. The exclusion criteria have to be defined unambiguously and must be rateable on a nominal scale marked 'probably complied' and 'not complied'. Exclusion criteria can be deduced from already available project targets and constraints, from superordinate or adjacent corporate partial strategies, or from further company-specific sources. In case a concept alternative does not fulfill any or all of the exclusion criteria, this concept alternative is not considered further for the following stages. Hence, pre-selecting ensures the compliance with important specifications and planning restrictions. On the other hand, it reduces the total expenditure for decision making without impairing the quality of the decision.

\subsection{Evaluating and Selecting Harmonization Concept Alternatives}

The holistic evaluation forms the core of the HDF that provides a method for the quantitative evaluation of the effects concerning the relevant target figures expected for a concept alternative. This includes effects concerning the singular PLM solutions involved as well as overall company-wide effects. The basis for this evaluation is a multi-criteria target system, which has been developed following the methodical approach of the Balanced Scorecard by Kaplan and Norton [9]. This target system is structured into the target areas of costs, benefits, and risks. The five PLM harmonization levels of PLM strategy, PLM processes, PLM methods, PLM systems, and PLM actors constitute the evaluation perspectives that must be considered simultaneously to achieve a holistic evaluation of the concept alternatives. The projection of the target areas on these evaluation perspectives comprises the depiction of the spectrum of potentially occurring effects of a concept alternative (cf. figure 4). Every evaluation perspective includes a generic set of evaluation criteria. Here criteria that are not directly monetarily quantifiable or partly subjective are also considered, apart from those that are directly monetarily quantifiable. 


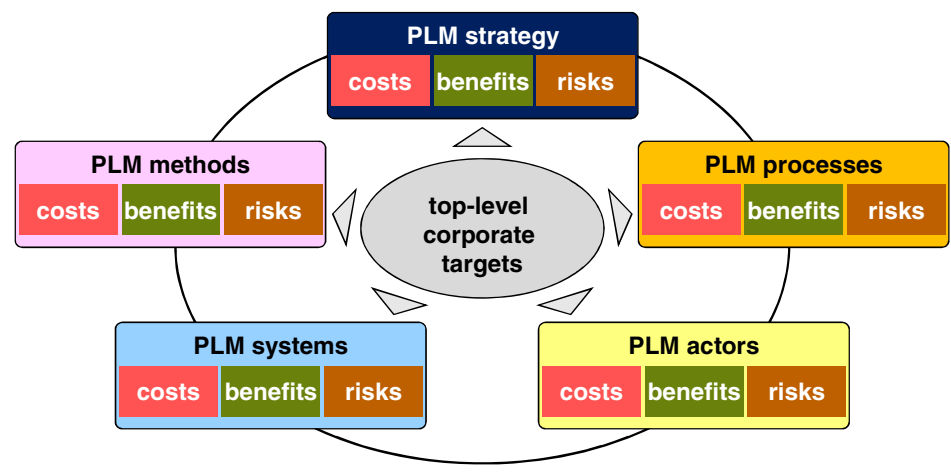

Fig. 4. Evaluation perspectives and target system areas

Criteria are considered directly monetarily quantifiable if their value can be directly and analytically explained and monetarily forecasted on the basis of documented objective information. In this case, the expected effects of a concept alternative can be evaluated at relatively high accuracy using simple mathematical terms (e.g. software license models and required quantity structure for the calculation of the expected licensing costs). If possible, criteria that cannot be directly monetarily evaluated are indirectly monetarily evaluated based on uncontroversial assumptions. As an example, the benefit of a faster part search can be estimated based on process time and staff costs. Many criteria that can have severe repercussions on a company's PLM success cannot be directly or indirectly evaluated monetarily, unless at extremely high expenditures due to complex multi-causal and long-term effects. This is particularly true for criteria of the target system areas of benefits and risks (e.g. strategic flexibility for the chosen vendor constellation). The consideration of such non-monetarilyquantifiable criteria constitutes an integral component of the developed evaluation method. The evaluation of non-monetarily-quantifiable criteria is made on the basis of assessments by experts and expert collectives as representatives of criteria-specific personalized knowledge and experiences [5].

Each of the approximately 100 evaluation criteria is specified by at least one Key Performance Indicator (KPI), which serves as a measurement for the value of the criteria. Each KPI is associated with a target system area and is classified according to its monetary quantifiability. Monetarily quantifiable criteria are depicted as absolute KPIs and measured on interval scales for the entire consideration period, generated by monetary calculation methods. They can be used simultaneously as a basis for a Total Costs of Ownership consideration. In contrast, non-monetarily quantifiable KPIs are defined by discrete, pre-defined maturity levels and measured on normalized ordinal scales or as continuous values on ratio scales. Within this work, extensive templates defining the generic target system have been developed including both types of KPIs for each evaluation perspective. To define a use case-specific evaluation model, these templates can be configured as to the focused PLM components and the use casespecific requirements. They follow a consistent structure as exemplified in figure 5 below. 


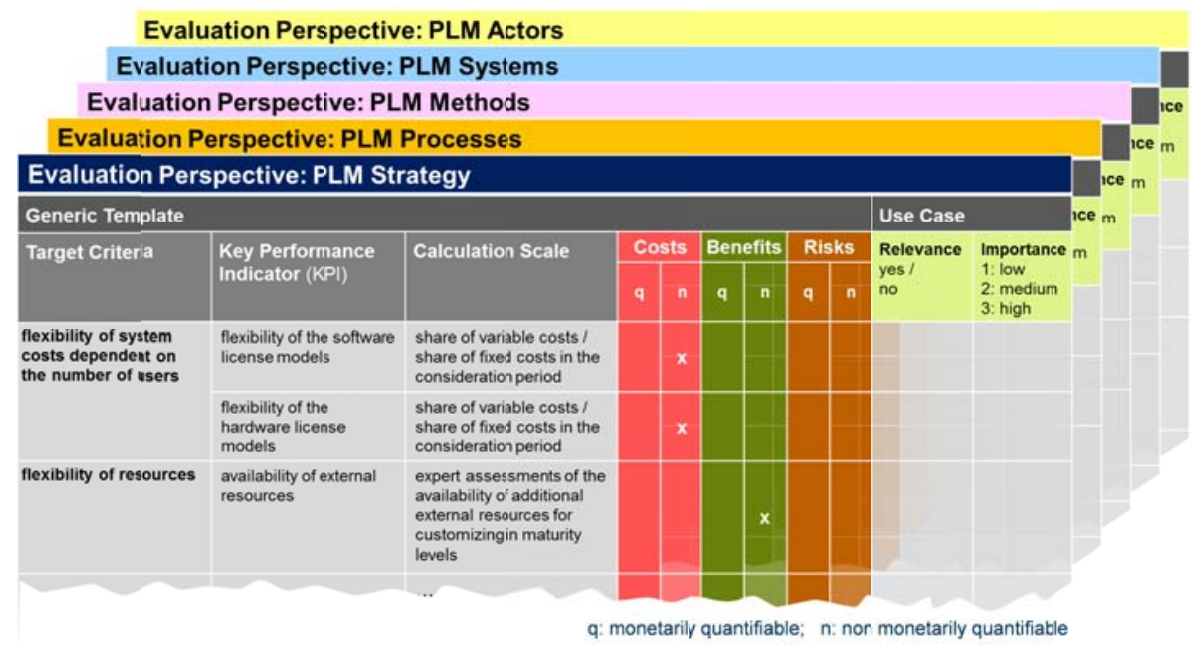

Fig. 5. Exemplary excerpt from the target system templates

For the evaluation perspective of 'PLM Strategy', the flexibility of resources and licensing models, the development of synergy potentials through homogenization, and the risks of implementation are important targets. For the evaluation perspective of 'PLM Processes', however, the process time, costs, quality, and complexity are crucial targets. The targets in the area of 'PLM Methods' include among others the coverage of the division-specific method requirements, the costs for method introductions and operations, as well as the homogenization of the methods applied. The number of the deployed IT systems and interfaces, the IT operating costs, the homogeneity of the data formats, and the support of the defined process and method demands with the existing IT systems are only some examples of targets of the evaluation perspective of 'PLM Systems'. The evaluation perspective of 'PLM Actors' includes different acceptance-related aims such as ensuring the required know-how and minimizing the effort of adjustment for PLM users, IT planners and administrators. Additionally, there are targets for the consequent consideration of the requirements and standards of the responsible top management.

Due to this evaluation method, the effects of each concept alternative are evaluated by representatives of all the company divisions involved, as well as company-internal and -external experts. Thus all aspects considered in a holistic use case-specific evaluation model are regarded for every company division involved as well as concerning overall effects. Furthermore, these aspects are weighted according to the agreed priorities of all decision makers. The evaluation result for any concept alternative can be illustrated by a harmonization scorecard that visualizes target conflicts of the use case-specific target system and provides information on directly and non-directly monetarily quantifiable effects. That way, the responsible managers can make substantiated, objectified, and transparent decisions as to the best harmonization concept alternative, in line with their preferences. Additionally, the transparent documentation of the evaluation results fosters the acceptance of the actors involved in the initiative. 


\section{$5 \quad$ Industrial Use Case}

The developed HDF has been implemented, validated, and extended within several industrial harmonization projects. The use case that is introduced in the following deals with the harmonization of the language variants of a system of PLM terms, which has been implemented in heterogeneous divisions of a multinational corporation in the machinery and plant industry. This use case was part of a company-wide PLM harmonization initiative that concerned more than 60 company divisions and was executed by a project team of more than 100 actors. In the initial situation, 24 PLM solutions had to be distinguished.

As a result of stage 1 (i.e. structuring PLM solutions), the system of terms for PLM object attributes (e.g. part names) that is assigned to the PLM method level was the only actively focused PLM component. In total, approximately 400,000 object attributes had to be considered, which contained different languages and different extents of translations. The following PLM components were found to be potentially passively affected:

- the PLM database concerning object attribute terms (level of PLM systems)

- the term representation in the graphical user interface (level of PLM systems)

- PLM user know-how related to the term system (level of PLM actors)

Stage 2 (i.e. defining the harmonization concept alternatives) yields that for the actively focused PLM component only basic harmonization approach III (merging) must be pursued. Since only one PLM component was actively focused, its variants likewise correspond to the harmonization concept alternatives (cf. fig. 6).

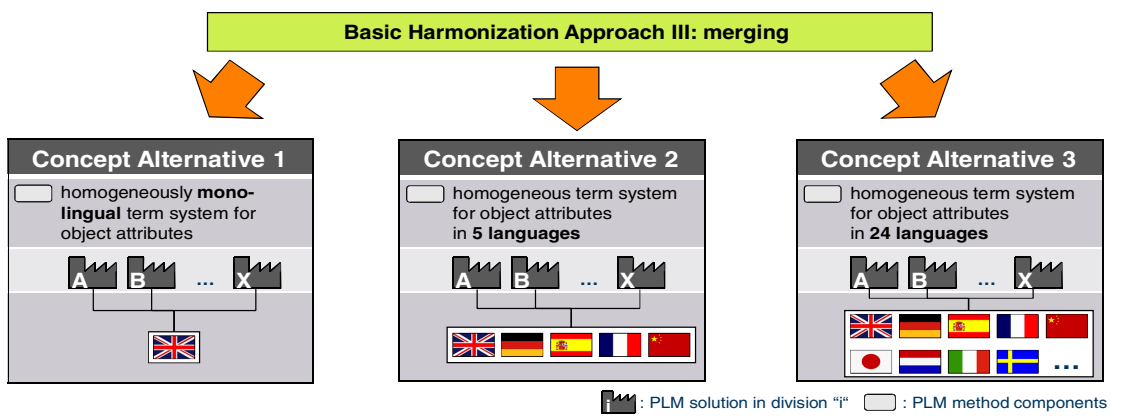

Fig. 6. Overview of the harmonization concept alternatives of the use case

No concept alternative could be excluded in the pre-selecting phase. Thus, a holistic evaluation of all of the three defined concept alternatives ensued in HDF phase 4. The harmonization scorecards of the concept alternatives exhibit vastly different characteristics based on the KPI measures (cf. fig. 7). In case of concept alternative No. 1 , very high actor-related costs (especially the costs of language training for PLM users) as well as high process- and actor-related risks dominate. Likewise, the costs of concept alternative No. 2 predominantly comprise actor-related costs and are altogether on a much lower level, slightly below the costs of concept alternative No. 3 . 


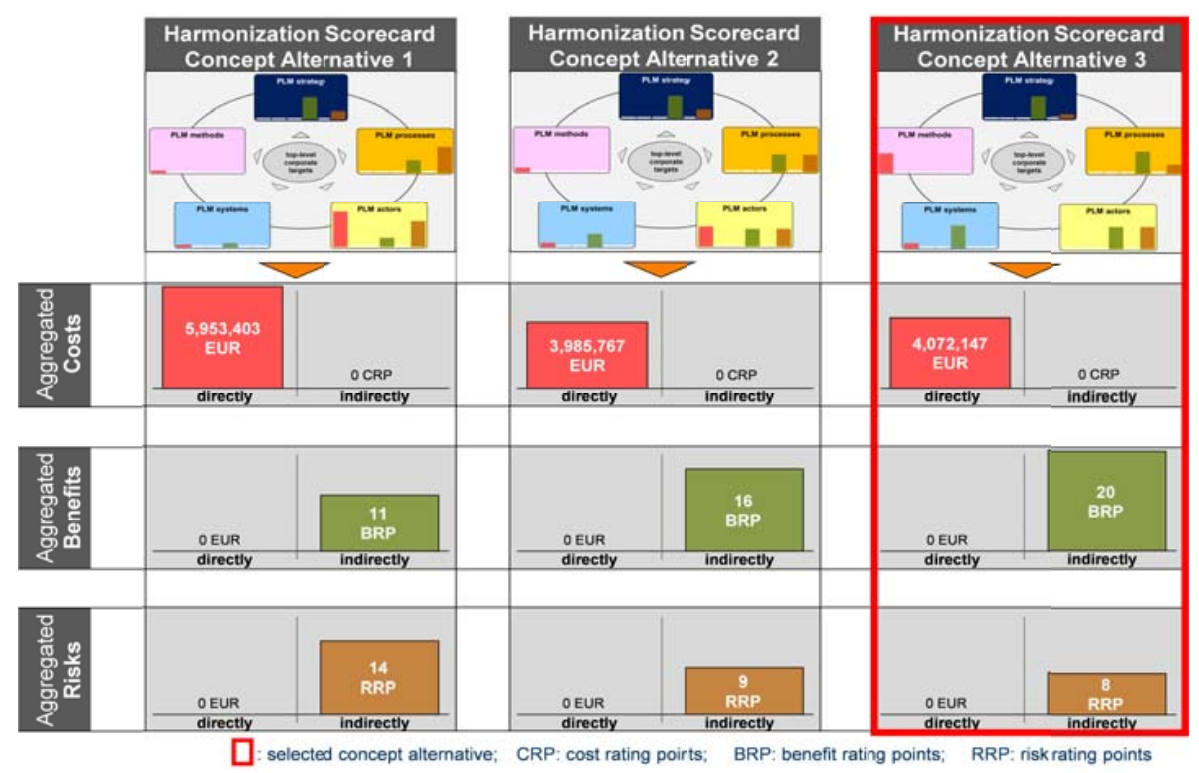

Fig. 7. Overview of the overall evaluations of the harmonization concept alternatives

The costs for concept alternative No. 3, however, mainly emerge from the PLM methods for the realization of the term system that comprises 24 languages in parallel. Apart from that, concept alternative No. 3 is characterized by the highest level of benefits (high process quality, low process time, standardized high availability of all object attributes) and the lowest level of risks of any alternative. In this use case, the collective of decision makers decided unanimously and supported by PLM experts that the generally high level of benefits and the low level of risks of concept alternative No. 3 far outweighed the lower costs for alternative No. 2 and therefore, alternative No. 3 had to be pursued.

\section{Conclusion and Outlook}

By conducting industrial PLM harmonization projects, project teams and decision makers often cannot cope with the task of determining the optimal harmonization concept alternative. In this case, they need to objectively consider the relevant constraints to all of the involved company divisions, top-level strategic targets, and the effects of different harmonization concept alternatives. Moreover, they pursue competing division-specific targets. The PLM Harmonization Decision Framework introduced in this paper has been developed to support decision makers and project teams with these challenges. Contrary to existing PLM procedure models, the approach does not address the introduction or optimization of singular PLM solutions, but the simultaneous and holistic design of several PLM solutions existing within a company. The approach has been developed in the context of several industrial projects carried out by the Chair of IT in Mechanical Engineering at Bochum Ruhr-University and has been illustrated by an industrial use case in this paper. In future, it will be integrated 
into a PLM harmonization framework that allows a holistic methodical support for PLM harmonization projects, including a specific procedure model.

\section{References}

1. Abramovici, M., Schindler, T.: Benefits of PLM in the Automotive Industry: results of a Benchmark Study. In: Proceedings of PLM 2010: The 7th International Conference on Product Lifecycle Management. CD-ROM. BIBA, Bremen (2010)

2. Abramovici, M., Göbel, J.: Methodical Approach to PLM Harmonization in Industrial Companies. ProductData Journal (2), 50-54 (2011), ProSTEP iViP, Darmstadt

3. Bitzer, M.: Entwicklung einer Methode zur Prozessorientierten Planung und Optimierung von Product Lifecycle Management Lösungen. Dissertation. Technical University of Kaiserslautern, Kaiserslautern (2008)

4. Black, B.: Building the future of manufacturing. In: Proceedings of the World Manufacturing Forum 2011. CD-ROM. World Manufacturing Forum, Como (2011)

5. Gigerenzer, G.: Bauch-Entscheidungen: Die Intelligenz des Unbewussten und die Macht der Intuition. Bertelsmann, Munich (2007)

6. Höfener, C.: Methode zur Bewertung des strategischen Nutzens von integriertem Produktdaten-Management (PDM). Dissertation. Technical University of Darmstadt, Darmstadt (1999)

7. Norm ISO 10303-239: Industrial automation systems and integration: product data representation and exchange, Part 239: application protocol: product lifecycle support. Beuth, Berlin (2005)

8. Kahlert, T.: Konzeption eines webbasierten Beratungs-Unterstützungs-Systems am Fallbeispiel einer PDM-Systemauswahl. Dissertation. Techical University of Berlin, Berlin (2000)

9. Kaplan, S., Norton, D.: Balanced Scorecard: Strategien erfolgreich umsetzen. SchäfferPoeschel, Stuttgart (1997)

10. Krause, L.: Methode zur Implementierung von integriertem Produktdatenmanagement (PDM). Dissertation. University of Bremen, Bremen (2002)

11. Pratt, M.J.: ISO 10303: the STEP standard for product data exchange, and its capabilities. In: Bouras, A. (ed.) International Journal of Product Lifecycle Management, vol. 1, pp. 86-94. Inderscience, Geneva (2005)

12. Rudolph, F., Dietrich, J.: Praktischer Einsatz von CAD- und EDM/PDM-Systemen. CADCAM Report, no. 5, pp. 58-61. Hoppenstedt, Darmstadt (2003)

13. Schabacker, M.: Benefit Evaluation of EDM/PDM Systems. In: Proceedings of ICE: 8th International Conference of Concurrent Enterprising. CD-ROM. Univ. of Nottingham, Nottingham (2002)

14. Schuh, G., Schlick, C., Schmitt, R., Lenders, M., Bender, D., Bohl, A., Gärtner, T., Hatfield, S., Müller, J., Mütze-Niewöhner, S.: Systemunabhängige Referenzprozesse für das PLM, Open Space Seminar, Aachen (2008)

15. Schmeja, M., Denger, A.: Future PLM : Ansätze für ein mitarbeiterzentriertes PLM. In: Proceedings of Product Life Live 2011, pp. 129-136. VDE, Berlin (2011)

16. Trippner, D.: Vorgehensmodell zum Management von Produktdaten in komplexen und dynamischen Produktentwicklungsprozessen. Dissertation. University of Karlsruhe, Karlsruhe (2002)

17. VDI-Gesellschaft Entwicklung Konstruktion Vertrieb: VDI 2219: Informationsverarbeitung in der Produktentwicklung: Einführung und Wirtschaftlichkeit von EDM/PDMSystemen. Beuth, Berlin (2002)

18. Verband Deutscher Maschinen- und Anlagenbau e.V. (VDMA): Leitfaden zur Erstellung eines unternehmensspezifischen PLM-Konzepts. VDMA, Frankfurt (2008) 